\title{
Hybrid NOMA-Based ACO-FBMC/OQAM for Next-Generation Indoor Optical Wireless Communications Using LiFi Technology
}

\author{
Hamis Hesham $^{1}$ and Tawfik Ismail ${ }^{1,2}$ \\ ${ }^{1}$ Wireless Intelligent Networks Center (WINC), Nile University, Giza 12677, Egypt \\ ${ }^{2}$ National Institute of Laser Enhanced Science, Cairo University, Giza 12613, Egypt
}

\begin{abstract}
The light fidelity ( $\mathrm{LiFi}$ ) has been successfully used to achieve high data transfer rates, high security, great availability, and low interference. In this paper, we propose a $\mathrm{LiFi}$ system consisting of a combination of non-orthogonal multi-access (NOMA), asymmetrically-clipped optical (ACO) and filter bank multicarrier (FBMC) techniques combined with offset quadrature amplitude modulation (OQAM). The paper also applies a $\mu$-law companding approach for a high peak to average power ratio (PAPR) reduction of the FBMC/OQAM scheme. The combination of NOMA, ACO-FBMC/OQAM, and $\mu$-law companding allows a significant increase in throughput and a significant reduction in non-served users. An appropriate algorithm is developed considering two scenarios, maximize the throughput and minimize the number of blocked (non-served) users. The results show that the throughput of the system can be increased by 1.8 compared to FBMC and OFDM. Furthermore, the proposed system reduces the number of blocked users below $10 \%$, while the system can provide $30 \%$ or $60 \%$ in case only the FBMC or OFDM is used, respectively.
\end{abstract}

Index Terms

Indoor optical communications, light fidelity, non-orthogonal multiple-access, filter bank multiple-access, companding.

\section{INTRODUCTION}

Visible light communication (VLC) is a form of the optical wireless communication (OWC) in which visible light is used to carry a signal. The VLC has been promoted as a complementary technology designed to reduce the overloads on conventional radio frequency (RF) technologies. A light fidelity (LiFi) utilizes the VLC channel to deliver wireless high-capacity transmission. The LiFi protocol is defined by IEEE 802.15.7 standard specification in 2011. The spectrum of light used by the LiFi system has a broad range of wavelengths from the infrared and visible to the most ultraviolet [1]. The LiFi also provides from hundred megabits to gigabits data rates for short and medium ranges [2]. Moreover, the LiFi technique offers new functionality to both existing and future services and allows new use cases for end-users.

Orthogonal frequency division multiplexing (OFDM) based LiFi scheme provides an opportunity for increasing the overall spectral efficiency, improving the energy consumption, and reducing transmission delay [3]. However, the conventional OFDM signal based on the Fourier Transform (FT) cannot be considered an effective solution for optical wireless communication because it does not meet the real and positive signal requirements. Modified variations of the conventional OFDM technique have been introduced to generate real and positive signals such as the asymmetrical clipped optical OFDM (ACO-OFDM), the dc-clipped optical OFDM (DCO-OFDM), and the odd-clipped optical OFDM (OCO-OFDM) [4] [5]. ACO-OFDM and DCOOFDM are applying Hermitian symmetry to optical carriers to generate a real OFDM signal. ACO-OFDM uses only the odd subcarriers to relay the data and clip the negative bits to zero. The DCO-OFDM process both positive and negative subcarriers by adding a DC component to the OFDM signal to produce positive results. In OCO-OFDM, Hermitian symmetry is replaced by the odd symmetry of Fourier transform, which improves its spectral efficiency for the real-modulation technique. Therefore, the ACO-OFDM is more efficient than DCO-OFDM, and DCO-OFDM has better spectral efficiency than ACO-OFDM. Moreover, the OCO-OFDM has the same spectral efficiency as the DCO-OFDM and the same power efficiency as the ACO-OFDM. However, the OCO-OFDM cannot be used for MPSK or MQAM modulation schemes. The most significant advantage of ACO-OFDM over other techniques is its ability to cope with extreme channel conditions without complex equalization filters by avoiding intersymbol interference (ISI).

Although, the OFDM provides easy bandwidth scalability by the configuration of the number of the subcarriers. OFDM simultaneously supports multiple users by assigning them specific subcarriers for intervals of time. This allows the access points to adjust the bandwidth usage according to the system requirements dynamically. However, a cyclic-prefix is inserted between adjacent OFDM in order to avoid the intercarrier interference (ICI) and to eliminate the high peak to average power ratio (PAPR), large side-lobes, and phase noise sensitivity [6]. An attractive multicarrier scheme based on the filter bank multicarrier (FBMC) has recently been proposed [7] [8]. The FBMC offers the potential for better spectral efficiency and spectral containment compared to traditional OFDM. Among various FBMC schemes, offset quadrature amplitude modulation (OQAM) is used with FBMC [9]. The FBMC/OQAM inherits the advantages of OFDM, such as excellent spectral efficiency 
and receiver sensitivity. Furthermore, the side-lobe suppression ratio of FBMC/OQAM is much higher than the conventional OFDM. The FBMC/OQAM can achieve smaller ICI by using well-designed pulse shapes that satisfy the perfect reconstruction conditions without using cyclic-prefix. This absence of cyclic-prefix creates a potential for improved bandwidth efficiency [10].

Non-orthogonal multiple access (NOMA) is one of the most promising access techniques in next-generation wireless communications for further significant capacity enhancement to accommodate traffic demand [11] [12]. It helps increase cell-edge throughput, enhances the cell-edge user experience, and gives massive communication by simultaneously supporting more uses [13]. The principle of NOMA includes non-orthogonal resource allocation among the users, which is required for separating the transmitted signals. It can perfectly yield a high spectral efficiency when the channel differential gains loss of path between users are large [14]. The NOMA schemes can be classified in general into two types: power-based and code-based. In power-based NOMA, the transmitter allocates different power coefficients for different users according to their channel conditions and achieves a high system performance. A successive interference cancellation (SIC) is a technique used by a NOMA receiver for efficient signal separation between two or more decoded signals arrived simultaneously [15]. In code-based NOMA, different users are allocated different codes and multiplexed over the same time-frequency resources [16].

This paper investigates a cooperative NOMA-based ACO-FBMC/OQAM over LiFi system. This investigation aims to address the resource allocation problem for multi-user downlinks to maximize the throughput and minimize the non-served users. To resolve the effect of PAPR, the $\mu$-law companding technique is presented under the Additive White Gaussian Noise (AWGN) channel.

The rest of the paper is structured as follows. Related works and preliminaries are presented in Section II and Section III, respectively. Section IV introduces the proposed hybrid access technique with ACO-FBMC/OQAM for downlinks to the LiFi network. A combined algorithm was proposed to optimize system throughput and minimize the number of non-serviced users in Section V. Simulation scenarios and results are discussed in Section VI. Finally, Section VII concludes the paper.

\section{RELATED WORKS}

ACO-OFDM is a widely accepted unipolar OFDM scheme proposed for OWC. The ACO-OFDM employs Hermitian symmetry to generate real bipolar OFDM signals. Furthermore, it utilizes only odd subcarriers to avoid a DC bias setup. As a result, all of the even subcarriers and half of the odd subcarriers are wasted. However, the ACO-OFDM scheme is shown to be more optically power-efficient and cost-effective for low/medium speed communications. In [17], the authors evaluated ACO-OFDM, DCO-OFDM, and asymmetrically clipped DC optical OFDM (ADO-OFDM). These techniques are evaluated and compared for different M-QAM modulation index in terms of bit error rate (BER). It is shown that ACO-OFDM, in general terms, presents a better performance compared to the conventional DCO-OFDM and ADO-OFDM schemes. In [18] a superposition-based LED nonlinearity reduction scheme for ACO-OFDM systems is proposed. In contrast, a non-redundant signal is superimposed with the ACO-OFDM signal to minimize the large amplitude signals and increase the signal symmetry clipped to zero. Furthermore, a threshold factor is introduced to limit the reduction amount of the large-amplitude signal. The numerical results of the proposed scheme show that it has the potential to outperform most conventional approaches in terms of BER. The results also show that the proposed scheme is energy-efficient since the large-amplitude signal reduction is equivalent to the symmetric signal enhancement without the need for additional power.

FBMC technique is a promising multicarrier modulation for future wireless communications. The FBMC technique overcomes the limitations of OFDM by adding generalized pulse shaping filters where no CP is required. Therefore, it offers a more effective use of radio resources. The BER performance for DCO-FBMC/OQAM was introduced in [19] over laser-based VLC link at $1.5 \mathrm{Gbit} / \mathrm{s}$. The BER performance of DCO-FBMC/OQAM and DCO-OFDM system under different received signal power are compared. The results show that using 64-QAM, the BER of DCO-FBMC/OQAM is lower than $10^{-4}$ compared to $10^{-3}$ when DCO-OFDM was used. ACO-FBMC/OQAM was first developed in [20]. The Interframe interference is eliminated using a proposed iterative receptive model. The BER performance over additive white Gaussian noise (AWGN) is studied with an 8 overlapping factor. The results show that the SNR is improved by $4 \mathrm{~dB}$ over the ACO-OFDM scheme due to the perfect rectangular pulse shaping which eliminates the out of band transmission. However, the authors did not study the effect of the VLC channel and ICI on system performance. To resolve the PAPR, a trellis-based selective mapping (TSLM) and $A$-law companding scheme is proposed in In [21]. The integrated TSLM and $A$-law companding scheme offers a lower BER than just $A$-law companding is used while the two techniques have the same computational complexity.

NOMA technique takes advantage of the power domain for multiple access, in which multiple users can share the same resources (time/frequency/code) instantaneously. In [22], a DCO-OFDM NOMA-based scheme for multiple access channels and broadcast channels in indoor VLC is proposed. The proposed scheme used a SIC-based decoding and joint maximum likelihood (JML) decoding to improve the performance of the VLC system in terms of BER and computational complexity. The results show that the proposed system gives less average BER when the received SNR exceeds $40 \mathrm{~dB}$. An OP-NOM scheme that uses the LED power/current linear dynamic range is developed in [23]. This scheme reduced the nonlinear distortion for low power signals, which improved the performance of the system in terms of BER when compared to conventional NOMA with the same driver circuit with a limited gain-bandwidth product. The feasibility of the NOMA-VLC with single carrier transmission is demonstrated experimentally in [24]. The effect of PAR on BER performance is investigated for both the 
downlink and the uplink. The proposed scheme that used single carrier transmission and frequency-domain SIC achieved a lower PAPR with a good balance between throughput and fairness of served users compared with NOMA-VLC with OFDM modulation.

In [25], the performance of the combined NOMA and VLC systems are characterized by a downlink guaranteed quality of service (QoS). In [26], the authors proposed a multi-user VLC network where mobile users with random position and vertical orientation are concurrently supported by proposing scheduling strategies and feedback mechanisms to achieve near-optimal summary performance. The authors in [27] applied a simplified gain ratio power allocation that obtains the channel gains by using a look-up table to ensure efficiency and fairness of resource distribution among the users. In [28], a multi-user VLC system with $4 \times 4$ multiple-input multiple-output (MIMO) was investigated. The gain ratio power allocation scheme was introduced to realize an effective and less complex power allocation scheme. The system sum rate of the VLC system increased by $40 \%$.

\section{PRELIMINARY AND DEFINITIONS}

\section{A. Resource Block}

This paper uses the concept of a resource block as the smallest distribution unit per user. The resource block is the lowest unit of resources that can be allocated to a user. The bandwidths defined by the 3GPP standard are 1.4,3,5,10,15, and 20 MHz. In Long-Term Evolution (LTE), one resource block is about $180 \mathrm{kHz}$, and a $20 \mathrm{MHz}$ leads about 100 blocks. If the modulation used is 64-QAM (6 bits per symbol), then the throughput of each resource block will be $1 \mathrm{Mbps}$.

\section{B. ACO-Filter Bank Multicarrier (ACO-FBMC)}

FBMC is an advanced version of OFDM, providing better bandwidth efficiency and greater resistance to narrowband noise effects. It integrates multiplexing and modulation operations by separating the wideband channel into several narrowband channels called subchannels. Furthermore, it overcomes the limitations of OFDM by introducing generalized pulse shaping filters that deliver a well-located subchannel in both the time and frequency domains. The FBMC uses the OQAM for individual sub-carrier to maximize the spectrum efficiency and lead to a higher data rate [29]. In FBMC/OQAM, multiple frames separated by a time shift of $T / 2$ are sent simultaneously, where $T$ is the FBMC/OQAM symbol period. Implicitly, the real component carries the first frame. In contrast, the second frame is carried by the imaginary component of the transmitted signal, representing the orthogonal phase between the simultaneously transmitted frames. The continuous-time baseband modulated signal type FBMC/OQAM can be expressed as [30]

$$
x(t)=\sum_{n=-\infty}^{\infty} \sum_{m=0}^{N_{c}-1} a_{m, n} g(t-n T / 2) e^{j 2 \pi m F t} e^{\theta m, n},
$$

where $N_{c}$ is the total number of subcarriers, $F$ is the frequency separation between adjacent subcarriers, $F=1 / T, a_{m, n}$ is the M-ary QAM symbol transmitted at time $n T$ and frequency $m F$, and $g(t)$ is the prototype filter impulse response. The $\theta m, n$ is a phase shift uses to guarantee the orthogonality between the in-phase and quadrature phase components which is given by

$$
\theta m, n=\frac{\pi}{2}(m+n)
$$

In order to use the FBMC/OQAM in the LiFi system, the time domain signal should be both real and positive; the asymmetrically-clipped optical (ACO) technique is employed to ensure that. In ACO-FBMC, only the odd subcarriers carry data symbols, while the even subcarriers are set to zeros, ensuring that the transmitted signal meets the non-negative requirement. The ACO modulated signal is expressed as follows [31]:

$$
Y[n]= \begin{cases}X\left[\frac{m-1}{2}\right], & \text { if } m \text { odd, } m<\frac{N_{c}}{2} \\ X\left[\frac{N_{c}-m-1}{2}\right], & \text { if } m \text { odd, } m>\frac{N_{c}}{2} \\ 0, & \text { otherwise }\end{cases}
$$

\section{Peak-to-Average Power Ratio (PAPR)}

One of the significant problems with the FBMC is the PAPR similar to OFDM and other multicarrier schemes. The PAPR results in nonlinearity and degradation in system performance due to LED chip overheating and signal clipping distortion. The PAPR is defined as the crest factor of the signal over average power. The mathematical definition of the PAPR denotes as [32]:

$$
\operatorname{PAPR}[Y(t)]=\frac{\max _{0 \leq t \leq T_{s}}|Y(t)|^{2}}{P_{\text {avg }}},
$$


where $Y(t)$ at the output of IFFT, $Y(t)=\operatorname{IFFT}\{X[k]\}, P_{\text {avg }}$ is the average power of signal $\{Y(t)\}$, and $T_{s}=N_{c} T$ is the duration of the symbol. Several PAPR reduction approaches for FBMC are presented such as $\mu$-law, A-law, clipping, non-linear companding, ton reservation, selective mapping (SLM) and partial transmit sequence (PTS) [33]. Among these approaches, the $\mu$-law PAPR reduction of FBMC/OQAM scheme will be used in this paper becouse it has low complexity and easy to be implemented.

\section{Mu-law companding technique}

Mu-law or $\mu$-law is an effective companding technique that can be used to reduce PAPR. In the $\mu$-law, the compressor characteristic is a linear form for low-level inputs and a logarithmic form for high-level inputs. $\mu$-law compression signal at the transmitter is expressed as:

$$
f(x)=x_{\max }(t) \times \operatorname{Sgn}(x(t)) \frac{\ln \left(1+\mu|x(t)| / x_{\max }(t)\right)}{\ln (1+\mu)},
$$

where $\mu$ is the compand parameter, $x(t)$ is the input instantaneous amplitude, and $x_{\max }(t)$ is the peak amplitude of $x(t)$.

\section{E. Non-orthogonal multiplexing access (NOMA)}

NOMA has been shown in the literature for further capacity enhancement and to maintain the future traffic demand [34] [35]. NOMA can yield the best performance over orthogonal multiple access (OMA) as weaker users reach a higher rate by considering stronger users as interference. The users with strong channel gain can first identify and delete the interference from weak users before decoding their signals. Therefore, this requires sufficient power to be assigned to the weaker users in order for such detection to be successful. At the transmitter side, the users are multiplexed in the power domain and are separated by SIC at the receiver side. Figure 1 presents the spectral occupancy of a two-users scenario. The horizontal axis indicates the bandwidth in terms of resource blocks (RBs), and the vertical axis is the power allocated for each resource. The achievable rate for the two users of the lower $(l)$ and higher $(h)$ channel gain are respectively given by [34]

$$
\begin{aligned}
& R^{l}=S \times B_{s} \log _{2}\left(1+\mathrm{SINR}^{l}\right), \\
& R^{h}=S \times B_{s} \log _{2}\left(1+\mathrm{SINR}^{h}\right),
\end{aligned}
$$

where $S$ is the total number of RBs and $B_{s}$ is the bandwidth of each RB. Additionally, The signal-to-interference-noise ratio (SINR) of the two users are given by

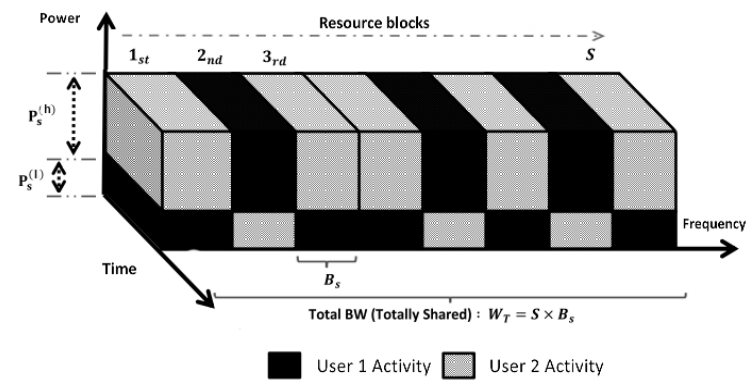

Fig. 1. Illustration of RB allocation for NOMA system

$$
\begin{aligned}
\operatorname{SINR}^{l} & =\frac{P_{s}^{l}\left|H^{l}\right|^{2}}{B_{s} N_{0}}, \\
\operatorname{SINR}^{h} & =\frac{P_{s}^{h}\left|H^{h}\right|^{2}}{B_{s} N_{0}},
\end{aligned}
$$

where $P_{s}^{l}=(1-\beta) P_{s}$ and $P_{s}^{h}=\beta P_{s}$ are the power allocated to lower and higher users, $\beta$ is the power ratio, $P_{s}$ is the allocated power for RB, $N_{0}$ is the noise power spectral density, $\left|H^{l}\right|^{2}$ and $\left|H^{h}\right|^{2}$ are the sum of all channel powers in the stronger and weaker users, respectively. These channel powers include the effect of fading, interference from a neighboring cell, and path loss while neglecting the co-channel interference and noise from pair users due to a perfect SIC mechanism with equalization technique is applied [36]. 


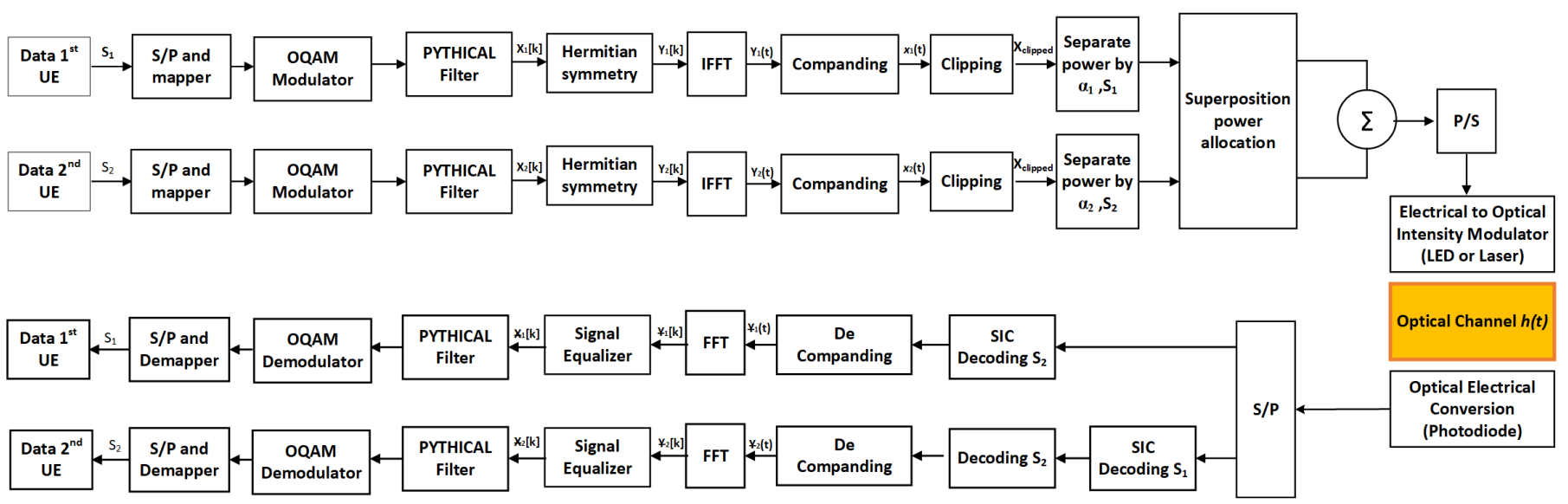

Fig. 2. Block diagram of hybrid NOMA-Based ACO-FBMC/OQAM system.

\section{F. VLC CHANNEL MODEL}

The VLC channel has two components, line-of-sight (LOS) and non-line-of-sight (NLOS). The LOS channel gain can be expressed as [37] [38]

$$
H_{\mathrm{LOS}}=\frac{(m+1) A_{\mathrm{PD}}}{2 \pi d_{u}^{2}} \cos ^{m}(\phi) T_{s}(\psi) g(\psi) \cos (\psi),
$$

where $A_{\mathrm{PD}}$ is the physical area of photodetector (PD), $m$ is the Lambertian order of the transmitter, $d_{u}$ is the distance between $\mathrm{LiFi} \mathrm{AP}$ and the user $u, \phi$ is the angle of irradiance, $\psi$ is the angle of incidenc, and $g_{c}$ is the optical concentrator gain, which is given by

$$
g(\psi)=\left\{\begin{array}{cr}
\frac{n^{2}}{\sin ^{2}(\psi)} & 0 \leq \psi \leq \psi_{f_{1 / 2}} \\
0, & \psi>\psi_{f_{1 / 2}}
\end{array},\right.
$$

where $n$ is the internal refractive index, and $\psi_{f_{1 / 2}}$ is the semi-angle of field of view (FOV) of PD. Additionally, the NLOS component is the superposition of all non-LOS components that are due to one or more reflections at the wall surfaces. The frequency dependence NLOS optical impulse response for a room corresponds to a first-order low-pass filter with transfer function is given by [38] [39]

$$
H_{\mathrm{NLOS}}(f)=\frac{\rho A_{\mathrm{PD}} \exp (j 2 \pi f \Delta T)}{A_{\text {room }}(1-\rho)\left(1+j f / f_{c}\right)},
$$

where $\rho$ is the wall reflectivity, $\Delta T$ is the delay between the LOS and diffused signals, $A_{\text {room }}$ is the area of the room, and $f_{c}$ is the cut-off frequency. The complete optical channel gain can be expressed as $H=H_{\mathrm{LOS}}+H_{\mathrm{NLOS}}$. The SINR for user (u) connected to LiFi AP $(\alpha)$ can be written as follows [40]:

$$
\operatorname{SINR}_{u, \alpha}=\frac{\left(H_{u, \alpha} P_{\mathrm{opt}} \Re\right)^{2}}{B_{s} N_{0}+\sum_{\xi \in \mathrm{AP}}\left(H_{u, \xi} P_{\mathrm{opt}} \Re\right)^{2}} ; \quad \alpha \neq \xi,
$$

where $P_{\text {opt }}$ is the average transmitted optical power of a LiFi AP, and $\Re$ is the responsivity of PD. Thus, the link data rate between user $(u)$ and AP $(\alpha)$ is calculated by the capacity lower bound, which can be written as [41]:

$$
R_{u, \alpha} \geq \frac{B_{s}}{2} \log _{2}\left(1+\frac{e}{2 \pi} \operatorname{SINR}_{u, \alpha}\right)
$$

\section{HYBRID NOMA-BASED ACO-FBMC/OQAM TECHNIQUE}

In this section, a multi-user hybrid NOMA-Based ACO-FBMC/OQAM over LiFi network is presented for indoor communication. Figure 2 illustrates the block diagrams of transmitter and receiver. At the transmitter, the M-QAM mapper separates real and imaginary parts of the input signal. Then, the imaginary parts are delayed by half of the symbol duration to realize OQAM conversion. The result from the OQAM mapper is processed by the physical prototype filter to create FBMC/OQAM symbols then processed by Hermitian symmetry and then carried by odd subcarriers. The generated symbols are then converted from the frequency domain to the time domain using IFFT. A $\mu$-law companding technique is applied to the IFFT signals to minimize the PAPR. Since only the odd frequency subcarriers are modulated, all of the intermodulation 
results from clipping falls on the even subcarriers and does not affect the data-carried by odd subcarriers. Because of the odd frequency and Hermitian constraint, there are only $N / 4$ input values are carried for $N$ points IFFT. Finally, both users with different power allocations, $\alpha_{u_{l}}$ and $\alpha_{u_{h}}$, are superposed in the power domain to yield NOMA signal over the VLC communication channel.

At the receiver, SIC is used to detect the user signal and treat the other as interference. In the SIC, the signals are ordered according to their SNR strengths, and the user decodes the stronger signal first, subtracting it from the combined signal and isolating the weaker. After successive isolating and decoding of the received signals, they are converted from the time domain symbols to the frequency domain using FFT. Afterward, the frequency domain signals are equalized and filtered by a physical prototype filter to remove the interference and noise received by the channel. Next, the transmitted symbols are demodulated and then demapping by the OQAM demapper, finally deinterleaves and decoded.

\section{PROPOSED COMBINED ALGORITHM}

In this section, we developed a combined algorithm, named positioning, clustering and resource allocation (PCRA), that performs the following steps: 1) determines the position of all UEs, 2) clusters the UEs based on geographical distribution under each AP to near and far UEs, 3) associates user pairing, and finally 4) distributes the resources (RBs and transmitted power) on the UEs in two scenarios maximizing the throughput or minimizing the blocked users. These steps are introduced in Algorithm 1, which is described in more detail as following.

\section{A. Positioning}

The positioning technique uses to determine the location of mobile user $u$ moving within the coverage area of AP $\alpha$. The VLC indoor positioning system is shown Figure 3. Several works have been successfully explored for accurate indoor positioning in VLC systems [42] [43]. The UE position $\left(x_{u}, y_{u}\right)$ is given by

$$
\begin{aligned}
& x_{u}=x_{\alpha} \pm d_{u, \alpha} \cos (\varphi), \\
& y_{u}=y_{\alpha} \pm \sqrt{d_{u, \alpha}^{2}-\left(x_{\alpha}-x_{u}\right)^{2}-h^{2}},
\end{aligned}
$$

where $x_{\alpha}$ and $y_{\alpha}$ is coordinate of $\mathrm{AP} \alpha, d_{u, \alpha}$ the distance between $\mathrm{AP} \alpha$ and $\mathrm{UE} u, \varphi$ is the incident angle to $\mathrm{PD}$, and $h$ is the vertical distance between the AP and UE [42].

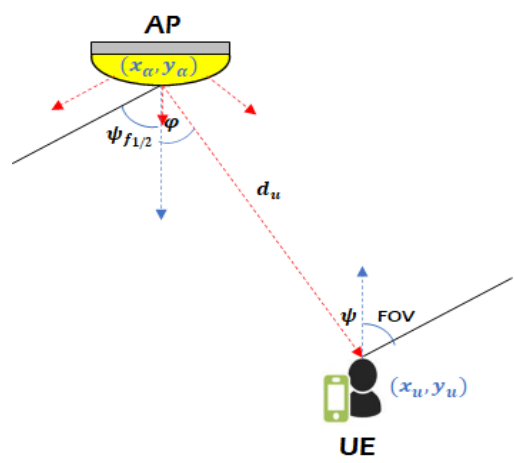

Fig. 3. Schematic diagram of the VLC-based positioning system

\section{B. Clustering and Association}

After allocating the position of all UEs within AP coverage, they will be sorted in ascending order based on their distance away from the AP and then divided them into two clusters (near and far), as shown in Figure 3. Subsequently, the two-user NOMA pair should be formed so that a minimum distance $\left(d_{\min }\right)$ separates the near and far users is a necessary condition to keep a minimum intra-cluster interference [44]. The distance between pair users in the two clusters, $\left(d_{\mathrm{up}}\right)$, is calculated by

$$
d_{\text {up }}=\sqrt{\left(x_{n}-x_{f}\right)^{2}+\left(y_{n}-y_{f}\right)^{2}} \geq d_{\min }
$$

where $x_{n}, y_{n}$ is the coordinates of near user, and $x_{f}, y_{f}$ is the coordinates of far user. 


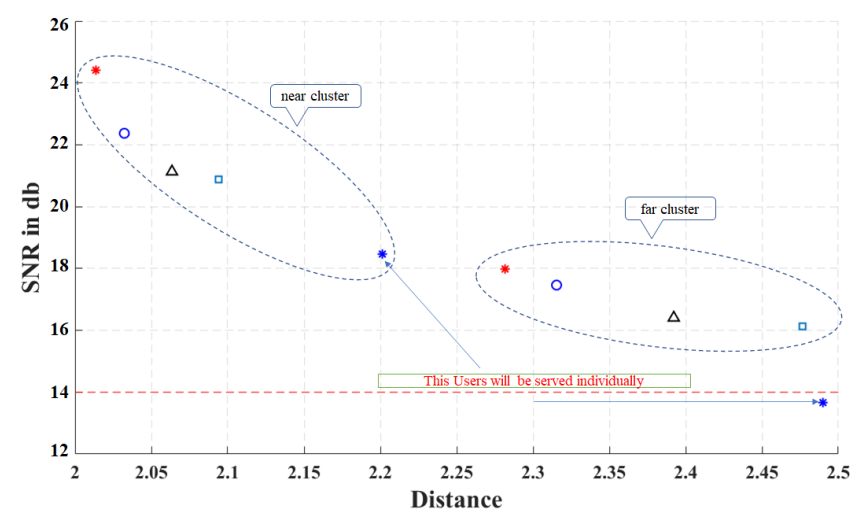

Fig. 4. Users distribution on two clusters

\section{Resource allocation}

The resource allocation mechanism should satisfy the quality of service (QoS) provisioning under the constraint of power and bandwidth (resource blocks) that maximizes the frequency reuse in the NOMA system. The Algorithm 1 has been developed on two scenarios 1) maximizing the overall system throughput and 2) minimizing the non-served users.

The following assumptions have been considered

- The users who did not satisfy the $d_{\min }$ condition will be excluded from the NOMA system and will be served individuals with lower priority,

- The power distributed factor between the two pairs (near and far) per RB is $\left(\beta P_{s}\right.$ and $\left.(1-\beta) P_{s}\right)$, respectively,

- Any user has SINR less than $14 \mathrm{~dB}$, the threshold of bit-error-rate (BER) $=10^{-3}$ in 64 -QAM, will not be served by the system.

\section{PERFORMANCE EVALUATION AND DISCUSSION}

This section discusses the numerical simulation and results for the proposed hybrid NOMA/FBMC system using two approaches, maximizing system throughput and minimizing the number of blocked (non-served) users. We considered a $16 \times 16 \times 4 \mathrm{~m}$ indoor space, which is entirely covered by $64 \mathrm{LiFi}$ APs. Table 1 shows the system parameters considered for simulation and the reported results as an average over 1000 iteration. The users are assumed to be uniformly distributed across the room, and they could require data rates up to $20 \mathrm{Mbps}$ follows a Poisson distribution.

TABLE I

SIMULATION PARAMETERS

\begin{tabular}{lcl}
\hline Parameter & Symbol & Value \\
\hline \hline Room Dimension & & $16 \times 16 \times 4 \mathrm{~m}$ \\
Access Point Spacing & & $2 \mathrm{~m}$ \\
Photo Diode Area & $A_{\mathrm{PD}}$ & $10^{-4} \mathrm{~m}^{2}$ \\
Vertical distance between UE and AP & $h$ & $3 \mathrm{~m}$ \\
Half-angle FOV & $\psi_{f_{1 / 2}}$ & $45^{0}$ \\
Gain of optical filter & $g_{c}$ & 1 \\
Semi-angle at half power & $\phi$ & $70^{\circ}$ \\
Refractive index of PD & $n$ & 1.47 \\
Noise power spectral density & $N_{0}$ & $10^{-21} A^{2} / \mathrm{Hz}$ \\
Downlink bandwidth & & $10 \mathrm{MHz}$ \\
IFFT length & & 512 \\
Optical Power & $P_{\mathrm{opt}}$ & $1 \mathrm{~W}$ \\
Number of subcarrier & & 256 \\
Frequency spacing & & $15 \times 10^{3} \mathrm{~Hz}$ \\
Overlapping factor & & 8 \\
Modulation index & $M$ & 64 \\
Number of FBMC symbols & & 64 \\
Number of resource block & $R_{\max }$ & 100 \\
Data rate per resource block & $R_{B}$ & $1 \mathrm{Mb} / \mathrm{s}$ \\
\hline
\end{tabular}

The optimized power distribution ratio between two paired users and the separation distance are evaluated in NOMA/FBMC models in order to select the best values of $\beta$ and $d_{\text {mim. }}$. Figure 5 illustrates the effect of changing the distance between the two paired users with SINR at various distributed power factors $(\beta=10 \%, 20 \%$, and $30 \%)$. As shown, the best configuration 


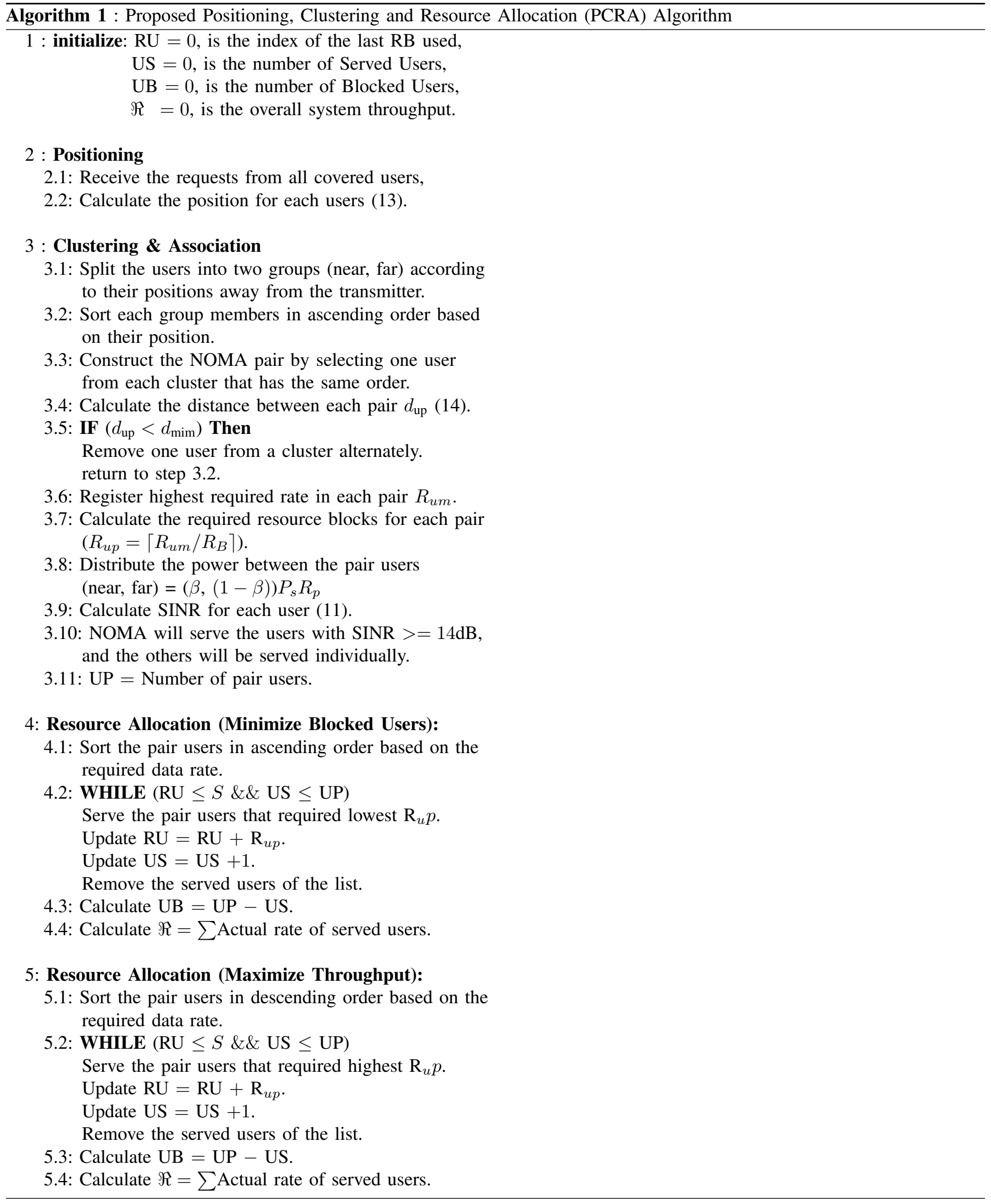

for achieving SINR greater than $14 \mathrm{~dB}$, which is required for BER $\leq 10^{-3}$, is to limit $\beta=20 \%$ and the separation distance to be greater than $2.5 \mathrm{~m}$.

Figure 6 shows the sum-rate versus the numbers of users with system resource blocks, $S$, equals 100 . Three different system 


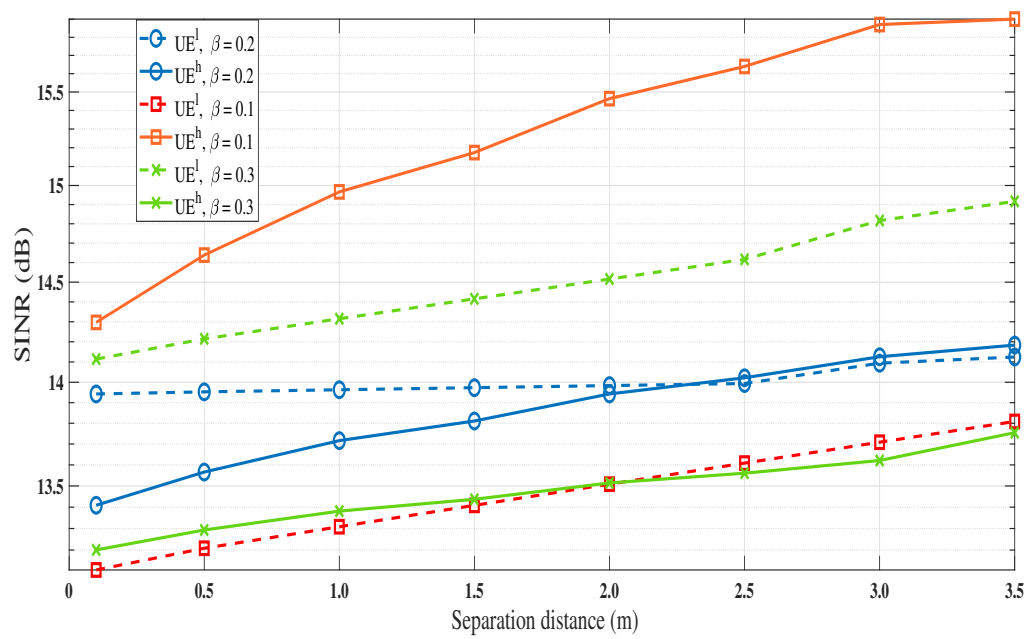

Fig. 5. SINR for $\mathrm{UE}^{l}$ and $\mathrm{UE}^{h}$ with different power ratio against separation distance $d_{\text {min }}$

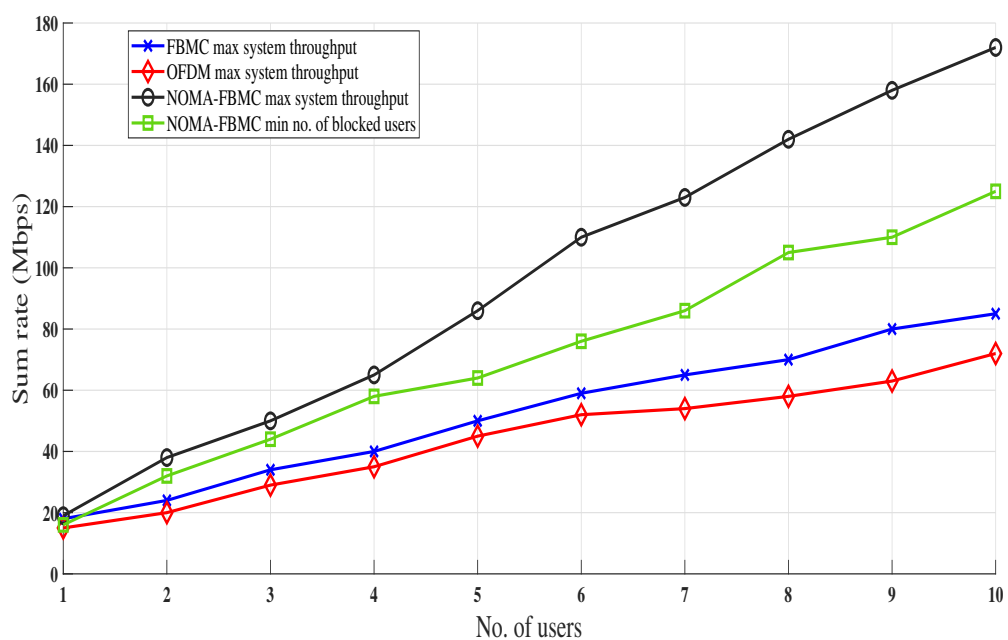

Fig. 6. Sum rate (Mbps) against No. of users with $P_{\mathrm{opt}}=1 \mathrm{~W}$, and $R_{B}=100$.

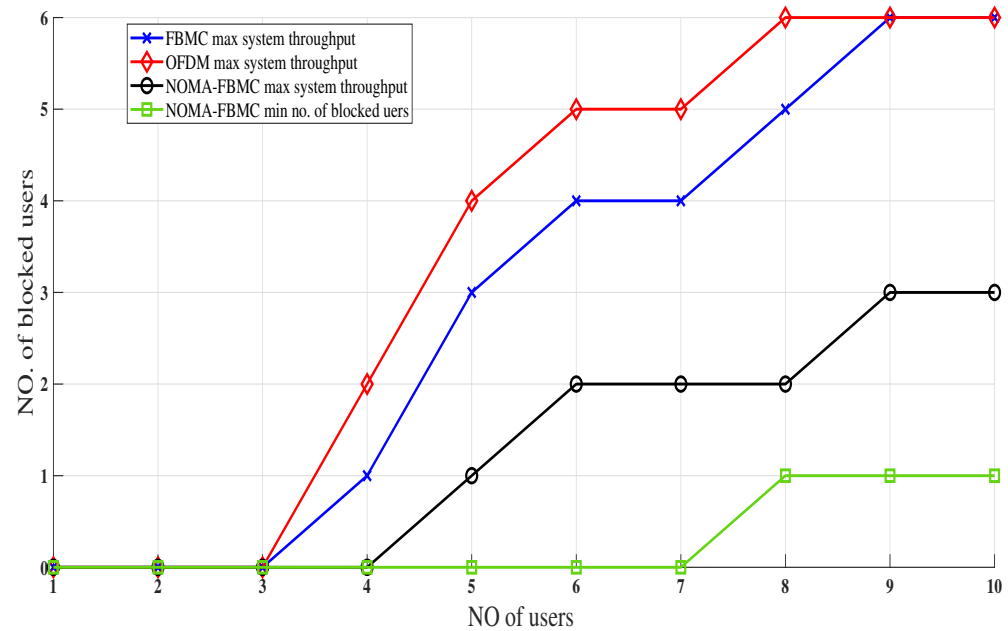

Fig. 7. No. of blocked uses against No. of users with $P_{\mathrm{opt}}=1 \mathrm{~W}$, and $R_{B}=100$. 
configurations are considered, FBMC only, OFDM only, hybrid NOMA/FBMC to maximize the overall system throughput. As shown, The proposed hybrid NOMA/FBMC system could significantly improve the throughput compared to when FBMC or OFDM is only used. In contrast, the system throughput could reach $170 \mathrm{Mbps}$ instead of $85 \mathrm{Mbps}$ at FBMC and $70 \mathrm{Mbps}$ at OFDM.

Finally, Figure 7 illustrates the comparison in terms of the number of blocked users versus the increasing number of users. The figure shows that, as the number of users increases, the number of blocked users also increases accordingly. However, the proposed algorithm, which a configuration that reduces the number of blocked users, achieves a better performance than other configurations. As shown, while the proposed algorithm could receive only $10 \%$ blocking, while the other three configurations provided $30 \%$ or $60 \%$ blocking for the proposed algorithm with high throughput and FBMC only, respectively.

\section{CONCLUSION}

In this paper, a hybrid structure that combines ACO, OQAM, FBMC, companding, and NOMA has been developed over the VLC communication channel. The proposed hybrid scheme allows two users simultaneously to be multiplexed over the shared RBs using a power allocation solution, enhancing the performance of the LiFi system. A resource allocation algorithm has been developed for two scenarios, maximizing the system throughput or minimizing the number of non-served users. The overall system, including ACO modulation, OQAM, OFDM, $\mu$-law, filters, optical modulation, are implemented using the MATLAB tools. The system is applied over the VLC channel considering the LoS and NLOS models. The developed algorithm showed that the proposed hybrid structure could increase the overall system throughput twice-times if only a FBMC was used and the case of OFDM. Furthermore, the proposed algorithm able to reduce the number of blocked users to less than $10 \%$ compared to FBMC with $30 \%$ and OFMC that has $60 \%$.

\section{DECLARATION}

We are enclosing herewith a manuscript entitled "Hybrid NOMA-Based ACO-FBMC/OQAM for Next-Generation Indoor Optical Wireless Communications Using LiFi Technology" for publication in Optical and Quantum Electronics Journal.

With the submission of this manuscript I would like to undertake that: - All authors of this research paper have directly participated in the planning, execution, or analysis of this study; - All authors of this paper have read and approved the final version submitted; - The contents of this manuscript have not been copyrighted or published previously; - The contents of this manuscript are not now under consideration for publication elsewhere; - The contents of this manuscript will not be copyrighted, submitted, or published elsewhere, while acceptance by the Journal is under consideration; - There are no directly related manuscripts or abstracts, published or unpublished, by any authors of this paper.

\section{CONFLICT OF INTEREST}

Manuscript title:

Hybrid NOMA-Based ACO-FBMC/OQAM for Next-Generation Indoor Optical Wireless Communications Using LiFi Technology.

The authors whose names are listed immediately below certify that they have NO affiliations with or involvement in any organization or entity with any financial interest (such as honor-aria; educational grants; participation in speakers' bureaus; membership, employment, consultancies, stock ownership, or other equity interest; and expert testimony or patent-licensing arrangements), or non-financial interest (such as personal or professional relationships, affiliations, knowledge or beliefs) in the subject matter or materials discussed in this manuscript.

Author names:

Hamis Hesham and Tawfik Ismail

\section{REFERENCES}

[1] X. Wu, M. D. Soltani, L. Zhou, M. Safari, and H. Haas, "Hybrid lifi and wifi networks: A survey," IEEE Communications Surveys Tutorials, pp. 1-1, 2021.

[2] H. Haas, L. Yin, Y. Wang, and C. Chen, "What is lifi?" Journal of Lightwave Technology, vol. 34, no. 6, pp. 1533-1544, 2016.

[3] A. J. Lowery, "Optical ofdm," in 2008 Conference on Lasers and Electro-Optics and 2008 Conference on Quantum Electronics and Laser Science, 2008, pp. $1-2$.

[4] A. Ibrahim, T. Ismail, K. F. Elsayed, M. S. Darweesh, and J. Prat, "Resource allocation and interference management techniques for ofdm-based vlc atto-cells," IEEE Access, vol. 8, pp. 127431-127 439, 2020.

[5] A. Ibrahim, T. Ismail, K. F. Elsayed, and M. S. Darweesh, "Odd clipping optical orthogonal frequency division multiplexing for vlc system," International Journal of Communication Systems, vol. 32, no. 16, pp. 1-13, 2019.

[6] M. Svaluto Moreolo, R. Munoz, and G. Junyent, "Novel power efficient optical ofdm based on hartley transform for intensity-modulated direct-detection systems," Journal of Lightwave Technology, vol. 28, no. 5, pp. 798-805, 2010.

[7] B. Farhang-Boroujeny and R. Kempter, "Multicarrier communication techniques for spectrum sensing and communication in cognitive radios," IEEE Communications Magazine, vol. 46, no. 4, pp. 80-85, 2008.

[8] S. N. Premnath, D. Wasden, S. K. Kasera, B. Farhang-Boroujeny, and N. Patwari, "Beyond ofdm: Best-effort dynamic spectrum access using filterbank multicarrier," in 2012 Fourth International Conference on Communication Systems and Networks (COMSNETS 2012), 2012, pp. 1-10.

[9] B. Saltzberg, "Performance of an efficient parallel data transmission system," IEEE Transactions on Communication Technology, vol. 15, no. 6, pp. 805-811, 1967. 
[10] A. Saljoghei, F. A. Gutiérrez, P. Perry, and L. P. Barry, "Filter bank multicarrier (fbmc) for long-reach intensity modulated optical access networks," Optics Communications, vol. 389, pp. 110-117, 2017.

[11] M. Obeed, A. M. Salhab, M. Alouini, and S. A. Zummo, “On optimizing vlc networks for downlink multi-user transmission: A survey," IEEE Communications Surveys Tutorials, vol. 21, no. 3, pp. 2947-2976, 2019.

[12] H. H. M. Mahmoud, A. Amer, and T. Ismail, "6g: A comprehensive survey on technologies, applications, challenges, and research problems," Transactions on Emerging Telecommunications Technologies, vol. e4233, pp. 1-14, 2021.

[13] L. Dai, B. Wang, Z. Ding, Z. Wang, S. Chen, and L. Hanzo, "A survey of non-orthogonal multiple access for 5g," IEEE Communications Surveys Tutorials, vol. 20, no. 3, pp. 2294-2323, 2018.

[14] A. Li, Y. Lan, X. Chen, and H. Jiang, "Non-orthogonal multiple access (noma) for future downlink radio access of 5g," China Communications, vol. 12, no. Supplement, pp. 28-37, 2015.

[15] H. Abumarshoud, H. Alshaer, and H. Haas, "Dynamic multiple access configuration in intelligent lifi attocellular access points," IEEE Access, vol. 7, pp. $62126-62141,2019$

[16] M. Aldababsa, M. Toka, S. Gökçeli, G. K. Kurt, and O. Kucur, "A tutorial on nonorthogonal multiple access for 5g and beyond," Wireless Communications and Mobile Computing, pp. 1-24, 2018.

[17] P. P. Játiva, C. A. Azurdia-Meza, M. R. Cañizares, D. Zabala-Blanco, and I. Soto, "Ber performance of ofdm-based visible light communication systems," in 2019 IEEE CHILEAN Conference on Electrical, Electronics Engineering, Information and Communication Technologies (CHILECON), 2019, pp. 1-6.

[18] Y. Jiang, M. Wang, X. Zhu, C. Liang, T. Wang, and S. Sun, "Superposition based nonlinearity mitigation for aco-ofdm optical wireless communications," IEEE Wireless Communications Letters, vol. 10, no. 3, pp. 469-473, 2021.

[19] R. Chen, K. Park, C. Shen, T. Khee Ng, B. S. Ooi, and M. Alouini, "Visible light communication using dc-biased optical filter bank multi-carrier modulation," in 2018 Global LIFI Congress (GLC), 2018, pp. 1-6.

[20] A. Ibrahim, J. Prat, and T. Ismail, "Asymmetrical clipping optical filter bank multi-carrier modulation scheme," in Optical and Quantum Electronics, vol. 53, 2021, pp. 1-12.

[21] X. Li, D. Wang, Z. Li, W. Bai, X. Hu, and R. Fu, "A hybrid tslm and $a$-law companding scheme for papr reduction in fbmc-oqam systems," in 2020 International Wireless Communications and Mobile Computing (IWCMC), 2020, pp. 1077-1081.

[22] T. Uday, A. Kumar, and L. Natarajan, "Noma for multiple access channel and broadcast channel in indoor vlc," IEEE Wireless Communications Letters, vol. 10 , no. 3 , pp. 609-613, 2021

[23] B. Lin, X. Tang, and Z. Ghassemlooy, "Optical power domain noma for visible light communications," IEEE Wireless Communications Letters, vol. 8, no. 4, pp. 1260-1263, 2019.

[24] B. Lin, X. Tang, Z. Ghassemlooy, Y. Li, M. Zhang, Y. Wu, and H. Li, "A noma scheme for visible light communications with single carrier transmission and frequency-domain successive interference cancellation," in Optik, vol. 193, 2019, pp. 445-450.

[25] L. Yin, X. Wu, and H. Haas, "On the performance of non-orthogonal multiple access in visible light communication," in 2015 IEEE 26th Annual International Symposium on Personal, Indoor, and Mobile Radio Communications (PIMRC), 2015, pp. 1354-1359.

[26] Y. Yapicı and I. Guvenc, "Non-orthogonal multiple access for mobile vlc networks with random receiver orientation," preprint arXiv, pp. 1-6, 2019.

[27] Q. Zhao, J. Jiang, Y. Wang, and J. Du, "A low complexity power allocation scheme for noma-based indoor vlc systems," Optics Communications, vol. 463, pp. 1-7, 2020.

[28] I. A. Elewah, F. Jasman, and S. Ng, "Performance enhancement for a nonorthogonal multiple access system using $4 \times 4$ multiple-input multiple-output visible-light communication," Optical Engineering, vol. 59, no. 12, pp. 1-11, 2020.

[29] J. Zhao, "Offset-qam multicarrier technology for optical systems and networks (invited)," in 2014 13th International Conference on Optical Communications and Networks (ICOCN), 2014, pp. 1-4.

[30] W. Khrouf, M. Siala, and F. Abdelkefi, "How much fbmc/oqam is better than fbmc/qam? a tentative response using the pops paradigm," Wireless Communications and Mobile Computing, vol. 2018, pp. 1-14, 2018.

[31] S. D. Dissanayake and J. Armstrong, "Comparison of aco-ofdm, dco-ofdm and ado-ofdm in im/dd systems," Journal of Lightwave Technology, vol. 31, no. 7, pp. 1063-1072, 2013.

[32] A. Mohammed, T. Ismail, A. Nassar, and H. Mostafa, "A novel companding technique to reduce high peak to average power ratio in ofdm systems," IEEE Access, vol. 9, pp. 35 217-35228, 2021.

[33] A. Agarwal and R. Sharma, "Review of different papr reduction techniques in fbmc-oqam system." Internet of Things and Big Data Applications-Springer, vol. 180, pp. 183-191, 2020.

[34] Z. Q. Al-Abbasi and D. K. C. So, "Resource allocation in non-orthogonal and hybrid multiple access system with proportional rate constraint," IEEE Transactions on Wireless Communications, vol. 16, no. 10, pp. 6309-6320, 2017.

[35] N. W. M. Thet, S. Khan, E. Arvas, and M. K. Özdemir, "Impact of mutual coupling on power-domain non-orthogonal multiple access (noma)," IEEE Access, vol. 8, pp. $188401-188414,2020$

[36] J. Datta and H.-P. Lin, "Detection of uplink noma systems using joint sic and cyclic fresh filtering," in 201827 th Wireless and Optical Communication Conference (WOCC), 2018, pp. 1-4.

[37] Z. Wang, Q. Wang, W. Huang, and Z. Xu, "Visible light communications: Modulation and signal processing," in Wiley-IEEE Press, 2018.

[38] R. Ahmad, M. D. Soltani, M. Safari, A. Srivastava, and A. Das, "Reinforcement learning based load balancing for hybrid lifi wifi networks," IEEE Access, vol. 8, pp. 132 273-132 284, 2020.

[39] H. Schulze, "Frequency-domain simulation of the indoor wireless optical communication channel," IEEE Transactions on Communications, vol. 64, no. 6, pp. 2551-2562, 2016.

[40] Y. Wang, D. A. Basnayaka, X. Wu, and H. Haas, "Optimization of load balancing in hybrid lifi/rf networks," IEEE Transactions on Communications, vol. 65 , no. 4 , pp. $1708-1720,2017$

[41] J. Wang, Q. Hu, J. Wang, M. Chen, and J. Wang, "Tight bounds on channel capacity for dimmable visible light communications," Journal of Lightwave Technology, vol. 31, no. 23, pp. 3771-3779, 2013.

[42] H. Hesham, T. Ismail, and M. S. Darweesh, "Indoor localization and movement prediction algorithms with light-fidelity," in 2020 22nd International Conference on Transparent Optical Networks (ICTON), 2020, pp. 1-4.

[43] G. Zayed, T. Ismail, and Y. Fahmy, "Visible light communications localization error enhancement using parameter relaxation," in 2020 16th International Computer Engineering Conference (ICENCO), 2020, pp. 191-196.

[44] S. Mounchili and S. Hamouda, "Efficient pairing distance for better radio capacity in noma systems," in 2020 4th International Conference on Advanced Systems and Emergent Technologies (ICASET), 2020, pp. 383-388. 